\title{
A Mechanics-based Model for Simulation and Control of Flexible Needle Insertion in Soft Tissue
}

\author{
Mohsen Khadem ${ }^{1}$, Bita Fallahi ${ }^{1}$, Carlos Rossa ${ }^{1}$, Ron S. Sloboda ${ }^{2}$, Nawaid Usmani ${ }^{2}$, Mahdi Tavakoli ${ }^{1}$
}

\begin{abstract}
In needle-based medical procedures, beveled-tip flexible needles are steered inside soft tissue with the aim of reaching pre-defined target locations. The efficiency of needlebased interventions depends on accurate control of the needle tip. This paper presents a comprehensive mechanics-based model for simulation of planar needle insertion in soft tissue. The proposed model for needle deflection is based on beam theory, works in real-time, and accepts the insertion velocity as an input that can later be used as a control command for needle steering. The model takes into account the effects of tissue deformation, needle-tissue friction, tissue cutting force, and needle bevel angle on needle deflection. Using a robot that inserts a flexible needle into a phantom tissue, various experiments are conducted to separately identify different subsets of the model parameters. The validity of the proposed model is verified by comparing the simulation results to the empirical data. The results demonstrate the accuracy of the proposed model in predicting the needle tip deflection for different insertion velocities.
\end{abstract}

\section{INTRODUCTION}

Percutaneous needle insertion is a minimally invasive medical procedure by which a surgeon inserts needles through the patient's skin to reach the inner body organs. Despite numerous advantages offered by needle-based interventions, their efficiency highly depends on accurate control of the needle tip in soft tissue. For instance, poor placement of radioactive isotopes in brachytherapy can cause long-term side effects (i.e. rectal or urinary damage) due to the disruption of neighboring tissues or organs. Various factors such as tissue deformation and needle deflection, which are in turn influenced by other factors such as insertion location, heading and velocity, can cause poor needle placement [1]. Considering the above and given the limited control exercised by a surgeon over the needle, prediction of the needle tip location and accurate steering of the needle during manual insertion is difficult. In this paper, a novel comprehensive mechanics-based model of needle-tissue interaction is presented that can be used for various purposes including the control of robotic-assisted needle insertion schemes.

\footnotetext{
*This work was supported by the Natural Sciences and Engineering Research Council (NSERC) of Canada under grant CHRP 446520, the Canadian Institutes of Health Research (CIHR) under grant CPG 127768 and the Alberta Innovates - Health Solutions (AIHS) under grant CRIO 201201232.

${ }^{1}$ M. Khadem, B. Fallahi, C. Rossa, PhD, and M. Tavakoli (Corresponding Author), $\mathrm{PhD}$, are with the Department of Electrical and Computer Engineering, University of Alberta, Edmonton, AB, T6G 2V4, Canada. \{mohsen.khadem, fallahi, rossa, mahdi.tavakoli\}@ualberta.ca

${ }^{2}$ R. S. Sloboda, PhD, FCCPM, and N. Usmani, MD, are with the Cross Cancer Institute and the Department of Oncology, University of Alberta, Edmonton, AB, T6G 1Z2, Canada. \{ron.sloboda, nawaid.usmani\} ealbertahealthservices.ca
}

\section{A. Background}

Researchers have been trying to enhance accuracy of needle insertions by developing models for simulating needle deflection in soft tissue. DiMaio et al. [2] and Goksel et al. [3] used Finite Element (FE) models of the tissue interacting with a rigid needle to find the needle tip position. In their models, they considered needles with symmetric tips. However, in needle-based interventions steerable flexible needles with beveled tips are used to enhance control over needle deflection. Using these needles a surgeon can control tip deflection by rotating the needle and changing the orientation of the bevel tip which causes the needle to bend in the opposite direction. These needles increase needle manipulability but finding an accurate model for relating the needle base manipulation to tip position becomes even more challenging. Webster et al. [4] developed a nonholonomic model for steering flexible bevel-tipped needles based on a kinematic bicycle model. Alterovitz et al. [5] presented a 2D FE model of needle insertion considering the effect of the tip bevel. Experimentally evaluated needle insertion forces are applied as boundary conditions on the elements of an FE tissue model to find tissue and needle deformation.

Other researchers have used beam theories to model needle deflection [6], [7]. From our group, Lehmann et al. [8] modeled an Euler-Bernoulli beam deflecting under static force distribution profiles to simulate needle insertion. Asadian $e t$ al. [9] developed a needle model comprised of two connected beams, one outside the tissue and the other inside. Misra et al. used an energy-based formulation for a beam that is in contact with a nonlinear hyperplastic tissue to simulate needle steering [10]. This model takes account of lateral and axial deflection of the needle, tissue deformation, forces at the tip of the needle and input force applied at the needle base. However, the effects of needle-tissue friction and needle insertion velocity were neglected.

In the preceding models, inputs may be the forces exerted on the needle as the result of tissue deformation as in [2], [5]. In this way, a tissue-dependent model is developed, i.e., the needle model interacts with a separate (experimental or theoretical) tissue model. Employing a comprehensive model of tissue can be very time consuming - more timeefficient ways to model tissue come at the expense of reduced accuracy [11], [3]. Most models are static and developed to correspond to a fixed set of inputs and model parameters [9] which should be identified each time for a pre-defined set of circumstances (e.g., for different insertion velocities) by conducting pre-surgery insertions [10] or using realtime measurements for online adaptive tuning of model parameters [12]. By not explicitly considering factors such as the insertion velocity as the model input, such approaches 
do not present sufficient control commands for real-time control of the needle tip position. To the best of the authors' knowledge, the nonholonomic bicycle-like model [4] is the only model that has insertion velocity as its input and is able to simulates deflection for given velocities. However, the model does not account for needle-tissue interaction along the needle shaft.

In conclusion, various inputs such as insertion velocity, needle tip rotation and needle base forces/torques can be used to control needle steering. However, a comprehensive dynamic or kinematic model is needed to relate these inputs to the system states and output (e.g needle shape and tip position). Otherwise in the static condition, the system is in balance means that the history of the input force or velocity on needle deflection is ignored and they cannot be used to control the needle deflection.

\section{B. Contribution}

In the current work, we have extended a beam theory to develop a novel mechanics-based dynamic model for needle insertion in soft tissue that considers velocity as an input. In section II, modeling techniques used in the analysis of vibration and dynamic response of beams under dynamic or static loads are employed to model the needle steering process. The proposed comprehensive model contains the effects of tissue deformation, needle-tissue friction, tissue cutting force, grid template used for positioning and guiding the needle, needle insertion velocity and bevel angle. In section III, using a needle-driving robot, experiments are conducted on a synthetic tissue and the accuracy of the model is verified.

The model enables one to use the insertion velocity as control command for needle steering. Since insertion velocity is easily tunable and accessible in needle steering, it is considered as the model input. Also, since computing the model output (needle deflection) given the model parameters (external forces applied on the needle) essentially amounts to solving the inverse dynamics of some robot-like dynamics, the model is computationally efficient and allows for realtime control of needle tip position in a robotic-assisted needle insertion scheme. All the model parameters represent mechanical characteristics of the needle-tissue system and can be separately measured. Consequently, there is no need for real-time feedback of measured system states for updating the model parameters.

\section{Mechanics-BAsed Model of NeEdle-Tissue INTERACTION}

\section{A. Needle-Tissue Modeling Approach}

In this section, a method is proposed to study the dynamics of a flexible needle as a cantilever beam undergoing various static and dynamic loads. The external loads represent the effects of the tissue and the grid template; the needle deflection is the dynamic response of the beam to these loads. The needle is modeled as a homogeneous, long and thin beam with a constant and symmetric cross section. Fig. 1 shows the proposed model of the flexible needle and its interaction with the soft tissue. To model the needle insertion, the beam is clamped to the local frame $(x y)$ that is moving
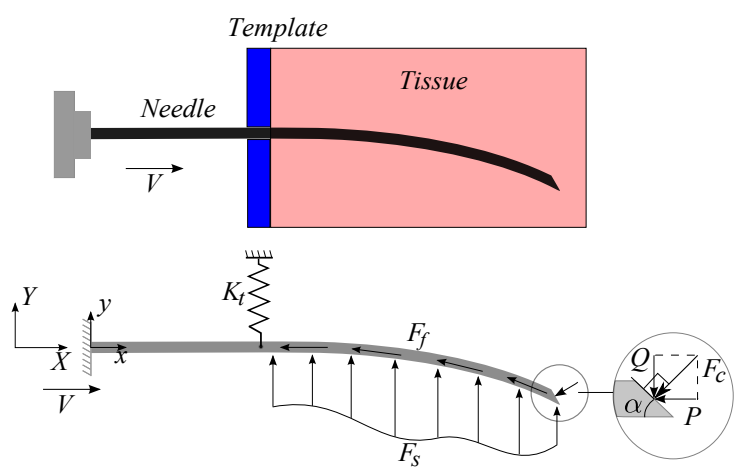

Fig. 1: Schematic of a bevel-tip needle inserted into a soft tissue.

along the $X$ direction in global non-moving frame $(X Y)$ with the velocity of $V$. Needle-template interaction is modeled using a stiff spring and needle-tissue interactions are modeled by axial and transverse distributed and concentrated loads. These forces are applied to the portion of the needle that is inside the tissue. As the needle is inserted into the tissue, the locations of these loads and the points of application of forces along the length of the needle change. The model considers four main elements of needle steering mechanics:

1) Bending of the needle during insertion: The needle is modeled as an Euler-Bernoulli beam that is translating along its longitudinal axis $(x)$. This is true if the beam's slenderness ratio (length/thickness) is much greater than 10 , which is the case for a brachytherapy needle. Mechanical characteristics of the needle encompass its Young's modulus of elasticity $E$, length $\ell$, density $\rho$, cross section area $A$, area moment of inertia $I$, and needle tip bevel angle $\alpha$.

2) Tissue cutting at the needle tip: As the needle is inserted into the tissue, a cutting force $F_{c}$, is applied to the needle in a direction perpendicular to the beveled tip. The transverse and axial component of $F_{c}$ are $Q$ and $P$, respectively, and are related by $Q=P / \tan (\alpha)$. It is typically assumed that the tip forces are initially zero and rapidly reach a constant value as the needle is inserted into the tissue [13] (see Fig. 1).

3) Needle-tissue interaction forces along the needle length: A force distribution $F_{S}$ is used to model tissue reaction forces as the result of its deformation caused by needle bending. Friction between the needle shaft and the tissue is modeled by an axially distributed load $F_{f}$ tangent to the needle shaft.

4) Needle-template interaction: The grid template used for initially positioning and subsequently guiding the needle acts as a rigid support and is modeled as a very stiff spring with stiffness $K_{t}$.

In our mathematical approach, instead of directly bringing the tissue and template into the dynamics equations, forces and moments produced by them are added to the system as external excitation forces. In this way we will be able to simplify the dynamics equation of the needle and bring in the effects of non-conservative forces such as friction.

\section{B. Dynamic Equations of Needle-Tissue Interaction}

In this section, we develop the governing equations of motion for the axially translating beam exposed to the external forces shown in Fig. 1. It is assumed that deflection of the needle is small compared to its length. We also presume needle bending is planar and in-plane axial bending and rotation of beam elements are negligible. Fig. 2 shows 


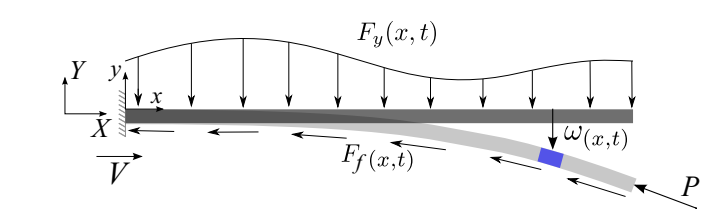

(a)

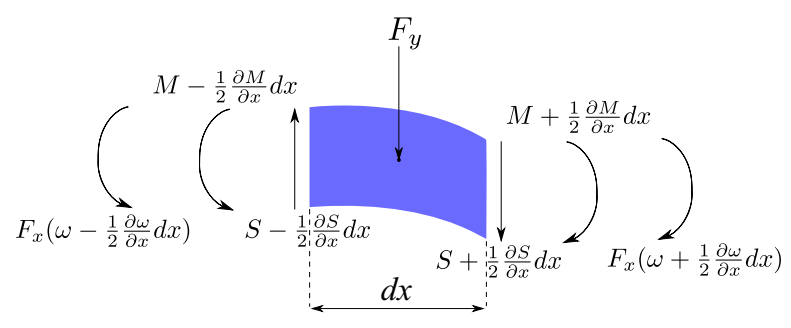

(b)

Fig. 2: Flexural behavior of straight beam in $x y$ plane under transverse and axial loads: (a) sketch of the system; (b) forces and moments acting on the length $d x$ of the beam

a small element of length $d x$ of the deformed beam. The (transverse) deflection of the needle is shown by $\omega(x, t)$, where $t$ is time and $x$ is the coordinate along the length of the unbent beam. $F_{y}(x, t)$ is an external load distribution and encapsulates all the transverse loads shown in Fig. 1 (i.e., $F_{s}, K_{t}$ and $\left.Q\right) . F_{f}(x, t)$ is the needle-tissue friction modeled as the uniformly distributed axial load. $P$ is a non-conservative follower force and always remains tangent to the beam at its tip location. $M$ is the internal bending moment, and $F_{x}$ and $S$ are the axial and shear forces acting on the small beam element, respectively. From here on, for simplicity, deflection, external load distribution, and friction force distribution are shown in the abridged form as $\omega, F_{y}$ and $F_{f}$, respectively.

The equilibrium equation for translations in the $y$-direction of the small element with length $d x$ of the beam is obtained by equating the inertial forces to the sum of exerted shear forces and external loads:

$$
\rho A d x\left(\frac{d^{2} \omega}{d t^{2}}\right)=\frac{\partial S}{\partial x} d x+F_{y} d x
$$

Note that since $\omega=\omega(x, t)$, we have $\frac{d \omega}{d t}=\frac{\partial \omega}{\partial t}+\frac{\partial \omega}{\partial x} \frac{d x}{d t}$. Now, (1) can be rewritten as

$$
\rho A\left(\frac{\partial^{2} \omega}{\partial t^{2}}+2 V \frac{\partial^{2} \omega}{\partial x \partial t}+V^{2} \frac{\partial^{2} \omega}{\partial x^{2}}+a \frac{\partial \omega}{\partial x}\right)=\frac{\partial S}{\partial x}+F_{y}
$$

where $a=d^{2} x / d t^{2}$ is the insertion acceleration and $V=$ $d x / d t$ is the insertion velocity. Surgeons tend to insert needles smoothly and avoid abrupt velocity changes, therefore we can neglect $a$.

The equilibrium equation for in-plane rotation of the length $d x$ is obtained by equating to zero the sum of the moment due to the shear forces acting at a distance $d x$, the internal bending moment, and the bending moment due to the axial forces. Note that it is assumed that the planar rotation of a small element of the beam is small and we can neglect axial and rotary inertia of the beam element. Thus, we have

$$
S d x+\frac{\partial M}{\partial x} d x+F_{x} \frac{\partial \omega}{\partial x} d x=0
$$

The bending moment is proportional to the curvature of the deflected beam. In fact, using Euler-Bernoulli beam theory, the bending moment is given by $M=E I \frac{\partial^{2} \omega}{\partial x^{2}}$ [14]. Also, the sum of axial resistive forces applied to element $d x$ is equal to $F_{x}=P+(\ell-x) F_{f}$ [15]. In (3), plugging in the above bending moment $M$ and axial force $F_{x}$ terms, taking a partial

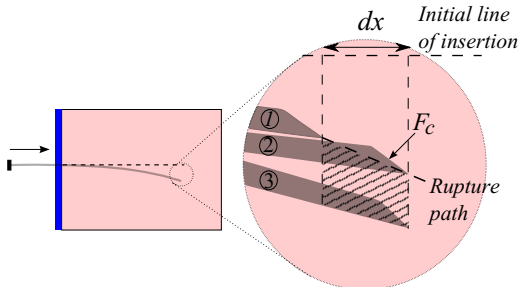

Fig. 3: Schematic of infinitesimal needle insertion: 1) needle in the beginning of insertion; 2) needle cuts through the tissue; 3) cutting force applied prependicular to the tip causes needle deflection and tissue deformation.

derivative with respect to $x$, and replacing for $\partial S / \partial x$ from (2) gives

$$
\rho A \ddot{\omega}+2 V \dot{\omega}^{\prime}+V^{2} \omega^{\prime \prime}+E I \omega^{\prime \prime \prime \prime}+P \omega^{\prime \prime}=F_{y}-\left[(\ell-x) F_{f} \omega^{\prime}\right]^{\prime}
$$

In (4), dot and prime denote differentiation with respect to $t$ and $x$, respectively. (4) is the partial differential equation (PDE) governing the dynamics of needle motion. $F_{y}$ and $F_{f}$ are transverse and axial force distributions containing all the forces applied by the tissue and the template except the axial component of cutting force. In section II-C we will model these forces.

\section{Modeling Needle-Tissue Interaction Forces}

In (4), $F_{y}$ contains all the transverse loads applied to the beam and comprises the forces applied due to the tissue compression, the template constraint and the transverse tip force. The transverse tip force $Q$ is a static concentrated force acting as a shear force at the tip of the beam. Other forces are moving loads acting over a specific length of the needle. As the needle is inserted, their point of application or width will change accordingly. To define the limits of the force profile applied to the length of the beam, we use unit step $(H)$ and Dirac delta $(\delta)$ functions defined in the global frame $X Y$. Now, we will assume a model for $F_{y}$ based on Fig. 1. $F_{y}$ can be defined as

$$
F_{y}=Q \delta_{\ell+V t}(X)+F_{s} H_{\ell}(X)+K_{t} \omega \delta_{\ell-\ell_{t}}(X)
$$

Note the shorthand notation $H_{x_{0}}(x)=H\left(x-x_{0}\right)$ and $\delta_{x_{0}}(x)=$ $\delta\left(x-x_{0}\right)$ describing unit step and Dirac delta functions shifted by the constant value $x_{0}$. Note that (4) is written in the non-inertial moving coordinates ( $x y)$ attached to the clamped side of the beam but the load distributions are written with respect to the global coordinates. The two frames are related by $x=X-V t, y=Y$. The tissue is placed at distance $\ell$ from the global frame and at $t=0$ the needle tip is touching the tissue skin. The coordinates of the needle base and tip as it is being inserted with velocity $V$ are $X=V t$ and $X=V t+\ell$, respectively. In (5), the first term is the transverse tip force - being a concentrated force fixed at the needle tip, it moves with constant velocity $V$ in the global frame as the needle is inserted, thus the term $\boldsymbol{\delta}_{\ell+V t}(X)$ has been used. The next term is the continuous distributed load exerted by the tissue and is activated as the needle tip enters the tissue (i.e., when $X>\ell$ ), thus involving the term $H_{\ell}(X)$. The last term is the force applied by the template, which is placed at a distance $\ell_{t}$ from the entry point, thus necessitating the term $\delta_{\ell-\ell_{t}}(X)$. The last two force profiles are fixed in the global frame. However, in the local frame, they seem to be shifting toward the base of the needle as the needle is inserted.

In $(5)$, the force distribution profile $\left(F_{S}\right)$ is used to model needle-tissue interaction. The magnitude of the approximated 
interaction forces are relative to the total deformation of tissue caused by needle deflection. Since the needle cuts a path through the tissue, only the tissue in between the rupture path and the current needle position is deformed. Fig. 3 shows the step-wise procedure used to investigate tissue deformation as the needle cuts through tissue. The hatched area shows the amount of tissue deformed as the result of a small insertion $d x$ of needle. Assuming that during an infinitesimal needle insertion the needle cuts through the tissue at a fixed angle and then it is bent as a result of the cutting force, the amount of deformed tissue in various steps are almost equal. Consequently, reaction forces applied to the needle are the same at each step and can be simulated with a uniformly distributed force profile.

Taking into consideration the viscoelastic behavior of the tissue, tissue-needle interaction force can be found as

$$
F_{s}=f_{s}+C \dot{\omega}
$$

where $f_{s}$ is the needle-tissue interaction force per unit length of the needle and is constant. $C$ is the coefficient of viscous damping for the tissue.

In (4), $F_{f}$ is the needle-tissue friction force modeled as a uniformly distributed tangential load. Let us use the simple friction model in [13] encompassing Coulomb and viscous forces per unit length of the needle:

$$
f=\mu_{c} \operatorname{sgn}(V)+\mu_{v} V
$$

In (7), $\mu_{c}$ and $\mu_{v}$ are the Coulomb and viscous friction coefficient, respectively. Now, we have $F_{f}$ as

$$
F_{f}=f H_{\ell}(X)
$$

Finally, by substituting (5), (6), (7) and (8) into (4), the PDE governing the dynamics of needle motion can be found as

$$
\begin{aligned}
& \rho A\left(\ddot{\omega}+2 V \dot{\omega}^{\prime}+V^{2} \omega^{\prime \prime}\right)+E I \omega^{\prime \prime \prime \prime}+P \omega^{\prime \prime}= \\
& Q \delta_{\ell+V t}(X)+f_{s} H_{\ell}(X)+C \dot{\omega} H_{\ell}(X)+ \\
& K_{t} \omega \delta_{\ell-\ell_{t}}(X)-f\left[H_{\ell}(X)(\ell-x) \omega^{\prime}\right]^{\prime}
\end{aligned}
$$

The clamped side of the needle is assumed to have no deflection and have a slope of zero. The tip of the needle is assumed to experience no shear force or bending moment. Also, small rotation of needle tip during needle insertion is neglected. Hence, we have the following set of boundary conditions:

$$
\omega(0, t)=0, \omega^{\prime}(0, t)=0, \omega^{\prime \prime}(l, t)=0, \omega^{\prime \prime \prime}(l, t)=0
$$

Note that instead of including $Q$ as a shear force in the boundary conditions, an appropriate forcing term is added as part of the transverse force distribution. The needle has no deflection at $t=0$ and starts from rest, therefore

$$
\omega(x, 0)=0, \dot{\omega}(x, 0)=0
$$

The PDE given by (9) cannot be solved using conventional methods such as separation of variables. Thus in the next section, it is discretized into a system of nonlinear ordinary differential equations (ODEs).

\section{Discretization of The Continuous PDE}

In this section, the continuous model in (9) is replaced by a discrete model using the assumed mode method [16]. The displacement field $\omega(x, t)$ is approximated by a linear combination of $n$ arbitrarily assumed functions $W_{i}(x)$ representing the first $n$ modes of vibration. These functions are trial shape functions which should satisfy the boundary conditions and be differentiable at least up to the highest order of the PDE. The displacement field is defined as

$$
\omega(x, t)=\sum_{i=1}^{n} \phi_{i}(t) W_{i}(x)
$$

where $\phi_{i}(t)(i=1, \ldots, n)$ are generalized coordinates or time functions expressing the deformation of the beam with respect to time and $n$ is the number of the assumed modes. Substituting (12) into (9) gives us the distribution of error of the assumed mode method, $e(x, t)$. At this point, we introduce the arbitrary function $U_{i}(x)$, and we minimize the projection of error on these functions:

$$
\int_{0}^{\ell} e(x, t) U_{i}(x) d x=0
$$

In (13), $U_{i}(x)$ does not need to satisfy any boundary or differentiability condition. However, convergence of the results of this approximation depends on a good choice of $U_{i}(x)$ [14]. Here we use a technique known as Galerkins method. Based on this method, a good choice for $U_{i}(x)$ is the assumed function $W_{i}(x)$ itself. Also by choosing the mode shapes of a homogenous clamped-free beam as assumed functions, we ensure that the assumed functions satisfy the boundary conditions and the results converge to the actual solution of PDE. The mode shapes for the clamped-free beam are given by [16]

$W_{i}(x)=\frac{1}{\zeta_{i}}\left\{-\frac{1}{\eta_{i}}\left[\frac{\cos \left(\theta_{i} x\right)}{\ell}-\frac{\cosh \left(\theta_{i} x\right)}{\ell}\right]+\frac{\sin \left(\theta_{i} x\right)}{\ell}-\frac{\sinh \left(\theta_{i} x\right)}{\ell}\right\}$

where $\theta_{i}(i=1, \ldots, n)$ is a dimensionless constant corresponding to different modes of vibration.

In this work, we will estimate the continuous model given in (9) using the first four modes. Values of the first four $\theta_{i}$ for a clamped-free beam are 1.857, 4.694, 7.855 and 10.996, respectively [16]. Also $\zeta$ and $\eta$ in (14) are given by

$$
\begin{gathered}
\zeta_{i}=\frac{\sin \theta_{i}+\sinh \theta_{i}}{\cos \theta_{i}+\cosh \theta_{i}} \\
\eta_{i}=-\zeta_{i}\left(\cos \theta_{i}-\cosh \theta_{i}\right)+\sin \theta_{i}-\sinh \theta_{i}
\end{gathered}
$$

Now, by taking the integral of (14) over the length of the needle, the discrete dynamic equation of the system can be obtained. The reference model for simulating deflection of a needle inserted in the tissue is given by

$\mathbf{M} \ddot{\phi}+\mathbf{G}(V) \dot{\phi}+\mathbf{K} \phi+\mathbf{B}(V) \phi+\mathbf{N} \phi=\mathbf{F}(\phi, V)+\mathbf{T}(\phi, V)+\mathbf{D}(\phi, V)+\mathbf{R}(\phi, V)$

By solving the above system of ODEs, time functions $\phi(t)$ can be found and deflection of the needle at each point $W_{i}(x)$ can be calculated by (12). The elements of matrices and vectors in (16) are given by

$$
\begin{gathered}
M_{i j}=\rho A \int_{0}^{\ell} W_{j}(x) W_{i}(x) d x, \quad G_{i j}=2 \rho A V \int_{0}^{\ell} W_{j}^{\prime}(x) W_{i}(x) d x, \\
K_{i j}=E I \int_{0}^{\ell} W_{j}^{\prime \prime \prime \prime}(x) W_{i}(x) d x, \quad B_{i j}=\rho A V^{2} \int_{0}^{\ell} W_{j}^{\prime \prime}(x) W_{i}(x) d x, \\
N_{i j}=P \int_{0}^{\ell} W_{j}^{\prime \prime}(x) W_{i}(x) d x, \quad F_{i}=Q W_{i}(\ell)+f_{s} \int_{\ell-V t}^{\ell} W_{i}(x) d x, \\
T_{i}=K_{t} W_{i}\left(\ell-\ell_{t}-V t\right) \sum_{j=1}^{n} \phi_{j}(t) W_{j}\left(\ell-\ell_{t}-V t\right), \\
D_{i}=C \int_{\ell-V t}^{\ell} W_{i}(x) \sum_{j=1}^{n} \dot{\phi}_{j}(t) W_{j}(x) d x, \\
R_{i}=-f\left[\int_{\ell-V t}^{\ell}(\ell-x) W_{i}(x) \sum_{j=1}^{n} \phi_{j}(t) W_{j}^{\prime \prime}(x) d x+\right. \\
\left.V t W_{i}(\ell-V t) \sum_{j=1}^{n} \phi_{j}(t) W_{j}^{\prime}(\ell-V t)-\int_{\ell-V t}^{\ell} W_{i}(x) \sum_{i=1}^{n} \phi_{j}(t) W_{j}^{\prime}(x) d x\right]
\end{gathered}
$$

The matrix $M$, which is associated with the kinetic energy 


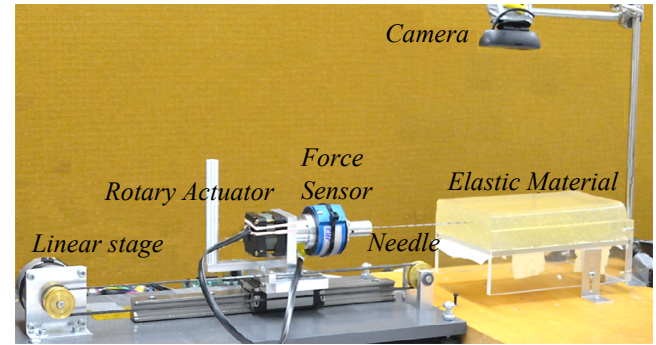

Fig. 4: Experimental setup used to steer a flexible needle though phantom tissue-The setup consists of a robotic system with two degrees of freedom (DOF), translational and rotational motion of needle. Forces and torques are measured at the needle base using a 6 DOF force sensor. Images of the needle inside tissue are recorded using a Logitech C270 webcam and is used to track needle position [8]

of the system, is positive definite. Due to using orthogonal eigen functions of a homogeneous clamped-free beam as the assumed modes, $M$ is also symmetric. $K$ corresponds to the elastic linear forces in the beam, it is also positive definite and symmetric. Both $M$ and $K$ are constant and time invariant. $G$ represents the gyroscopic forces and $B$ corresponds to inertial forces applied to the beam elements as the result of axial translation. $G$ and $B$ depend on the insertion velocity and appear in (17) because the vibration of the beam is described with respect to non-inertial moving coordinates. $N$ contains the effects of non-conservative forces which give rise to self-excitation of the beam. The terms on the right side of the system of ODEs in (16) correspond to the external excitation forces and moments and they are all functions of $V$.

\section{EXPERIMENTAL VALIDATION}

The parameters of the model given by (16) are the friction force $(f)$, tip forces $(P$ and $Q)$, needle-tissue interaction force $\left(f_{s}\right)$ and tissue viscous damping coefficient $(C)$. In the following section, a set of experiments is proposed to identify values of model parameters. In addition, needle deflection predicted by the model is compared to that during actual needle insertion into phantom tissue to verify the model accuracy.

\section{A. Model Calibration and Measurement Procedures}

In order to perform needle insertion into soft tissue, the setup introduced in [8] is used (Fig. 4). The needle used for the experiments is a standard 18-gauge brachytherapy needle (Best Medical International Inc., Springfield, VA, USA) made of stainless steel, with an outer diameter of 1.27 $\mathrm{mm}$ and an inner diameter of $1 \mathrm{~mm}$. The needle bevel angle is $20^{\circ}$. The phantom tissue is made of $80 \%$ liquid plastic and $20 \%$ plastic softener (M-F Manufacturing Co., Fort Worth, TX, USA) The tissue has a Young modulus $E$ of $28 \mathrm{kPa}$.

In order to measure elements of cutting force $(Q$ and $P$ ) and the friction force $(f)$, the method proposed in [13] is used. A small needle is inserted into a small piece of phantom tissue at different insertion velocities. The insertion is continued while the needle tip is outside the tissue. During the insertion the axial forces are measured using the force sensor placed at the needle base. In the first interval in which the needle tip is inside the tissue, the insertion force equals the force to overcome friction plus the axial element of cutting force $P$ (we neglect inertial forces). In the next phase where the needle tip is outside, the cutting force applied at the tip becomes zero and the measured axial force corresponds only to the friction force. Therefore using the measured forces in the second phase we can identify friction force. This data is fitted to (7) to identify the Coulomb $\left(\mu_{c}\right)$ and viscous friction coefficients $\left(\mu_{v}\right)$. Moreover, by subtracting the friction force from the measured forces in the first interval, the axial element of cutting force $(P)$ can be calculated. Finally, the transverse element of cutting force can be calculated by $Q=P / \tan (\alpha)$, where $\alpha$ is the bevel angle. In this experiment, a small needle with a length of 80 $\mathrm{mm}$ was robotically inserted into the synthetic tissue with a length of $40 \mathrm{~mm}$ at the insertion velocities of $5,10,20$ and $30 \mathrm{~mm} / \mathrm{sec}$. The shortened needle is used to minimize its deflection and the consequent effect on measured axial forces.

In the next step, experiments are conducted on the phantom tissue to identify $f_{s}$ and $C$. The needle is robotically inserted into the phantom tissue at three different velocities $(5,20,40$ $\mathrm{mm} / \mathrm{sec}$ ). By performing a multi-objective optimization, the experimental data for the three insertions are simultaneously fitted to the nonlinear system of ODEs defined by (16) and the two aforementioned parameters are identified. Genetic algorithm is used to minimize the residual error between the experimental data points and the predicted ones [17]. The magnitude of the constant force profile $f_{s}$ and damping coefficient of tissue per unit length of the needle $C$ are calculated to be $2.3 \mathrm{~N} / \mathrm{m}$ and $796 \mathrm{~N} . \mathrm{sec} / \mathrm{m}^{2}$ with $90 \%$ confidence intervals of \pm 0.109 and \pm 6.374 , respectively. Estimated model parameters and mechanical properties of the needle provided by the manufacturer are given in the first and the second row of Table I, respectively.

TABLE I: Values of model parameters

\begin{tabular}{cccccc}
\hline$f_{s}[\mathrm{~N} / \mathrm{m}]$ & $C\left[\mathrm{~N} . \mathrm{sec} / \mathrm{m}^{2}\right]$ & $\mu_{c}[\mathrm{~N} / \mathrm{m}]$ & $\mu_{v}\left[\mathrm{~N} . \mathrm{sec} / \mathrm{m}^{2}\right]$ & $P[\mathrm{~N}]$ & $Q[\mathrm{~N}]$ \\
\hline 2.3 & 796 & 5.1 & $1.6 \times 10^{2}$ & 0.088 & 0.244 \\
\hline$K_{t}[\mathrm{~N} / \mathrm{m}]$ & $\ell[\mathrm{m}]$ & $\rho\left[\mathrm{Kg} / \mathrm{m}^{3}\right]$ & $A\left[\mathrm{~m}^{2}\right]$ & $I\left[\mathrm{~N} . \mathrm{sec} / \mathrm{m}^{2}\right]$ & $E_{\text {Needle }}[\mathrm{GPa}]$ \\
\hline $10^{4}$ & 0.185 & 8030 & $4.81 \times 10^{-7}$ & $7.75 \times 10^{-14}$ & 200 \\
\hline
\end{tabular}

Note that in our experiments, the grid template is not used. However, the entry point into the tissue experiences negligible deformation during the insertion. Thus, the tissue surface effectively acts as a template. In order to model the rigid support, the stiffness of the template is arbitrarily chosen to be very large $\left(10^{4} \mathrm{~N} / \mathrm{m}\right)$ and it is placed at the point of entry $\left(\ell_{t}=0\right)$.

\section{B. Performance Assessment}

In this section, the parameters of Table I are imported into the model and needle insertion into the soft tissue is simulated for five different velocities $(5,10,20,30,40$ $\mathrm{mm} / \mathrm{sec}$ ). Also, using the robotic setup, the needle is inserted to a depth of $140 \mathrm{~mm}$ into the soft tissue phantom at the

TABLE II: Results of $140 \mathrm{~mm}$ insertion of the needle. Final tip position in the experiments $\omega_{\exp }(\ell)$, final tip position in the simulation $\omega_{\text {sim }}(\ell)$, maximum tip error $e_{\max }$, the root-mean-squared error of the whole insertion process $R M S E$, and standard deviation of final tip position $\sigma$ are listed

\begin{tabular}{|c|c|c|c|c|c|}
\hline$V[\mathrm{~mm} / \mathrm{sec}]$ & $\omega_{\exp }(\ell)[\mathrm{mm}]$ & $\omega_{\text {sim }}(\ell)[\mathrm{mm}]$ & $e_{\max }[\mathrm{mm}]$ & $R M S E[\mathrm{~mm}]$ & $\sigma[\mathrm{mm}]$ \\
\hline 5 & 11.17 & 11.06 & 0.66 & 0.29 & 0.75 \\
\hline 10 & 11.67 & 11.97 & 0.39 & 0.19 & 0.89 \\
\hline 20 & 12.66 & 13.27 & 0.61 & 0.32 & 0.60 \\
\hline 30 & 13.69 & 14.14 & 0.52 & 0.30 & 0.61 \\
\hline 40 & 14.54 & 14.76 & 0.57 & 0.38 & 0.58 \\
\hline
\end{tabular}




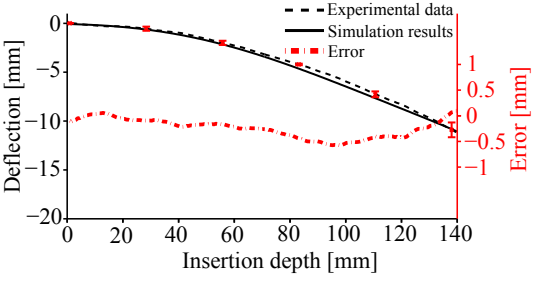

(a) $5 \mathrm{~mm} / \mathrm{sec}$ insertion velocity

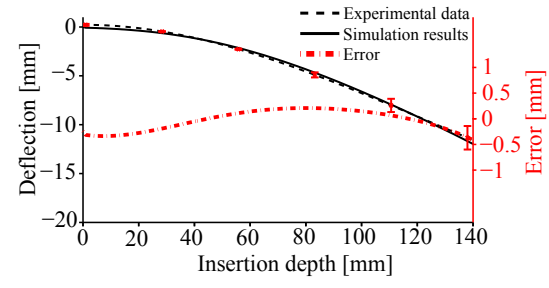

(b) $10 \mathrm{~mm} / \mathrm{sec}$ insertion velocity

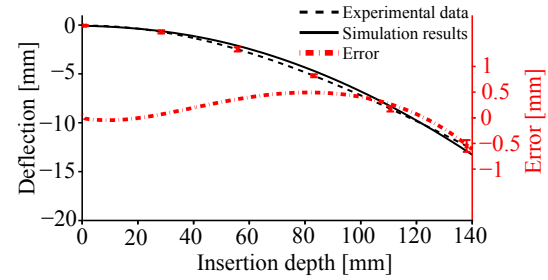

(c) $20 \mathrm{~mm} / \mathrm{sec}$ insertion velocity

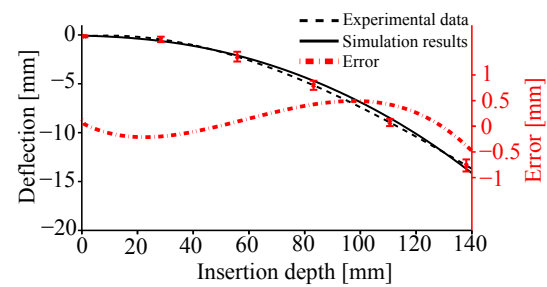

(d) $30 \mathrm{~mm} / \mathrm{sec}$ insertion velocity

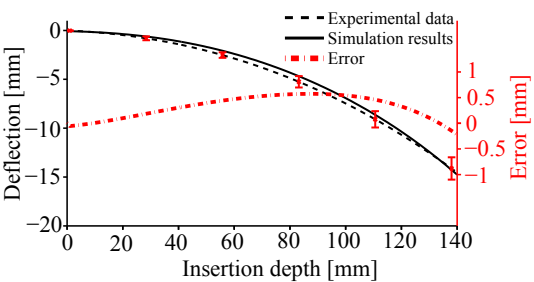

(e) $40 \mathrm{~mm} / \mathrm{sec}$ insertion velocity

Fig. 5: Comparison of simulation results from the identified model and the experimental data for needle steering at velocities of (a) $5 \mathrm{~mm} / \mathrm{sec}$, (b) $10 \mathrm{~mm} / \mathrm{sec}$, (c) $20 \mathrm{~mm} / \mathrm{sec}$, (d) $30 \mathrm{~mm} / \mathrm{sec}$, and (e) $40 \mathrm{~mm} / \mathrm{sec}$

same velocities (five trials for each velocity). Fig. 5 shows the simulated tip deflection compared to the average of five experimental insertions. The results are summarized in Table II. The maximum error in predicting the tip position is 0.66 $\mathrm{mm}$ at the depth of $95 \mathrm{~mm}$ and an insertion velocity of 5 $\mathrm{mm} / \mathrm{sec}$. Also, the results show that the maximum root-meansquared error (RMSE) of the model in predicting tip position is $0.38 \mathrm{~mm}$ for the insertion velocity of $40 \mathrm{~mm} / \mathrm{sec}$.

\section{CONCluding Remarks}

In this work a mechanics-based model of flexible needletissue interaction is developed to simulate needle insertion into tissue. Needle insertion velocity is the input to this model and can be used as a control command for roboticallycontrolled needle steering. Experimental results from robotic insertion of a brachytherapy needle into phantom tissue demonstrated the accuracy of the model and its sensitivity to the insertion velocity as the model input. In the collected experimental data, the maximum error of the model in predicting the needle tip position was $0.66 \mathrm{~mm}$ at the depth of $95 \mathrm{~mm}$. The proposed model can be used to provide the surgeon with real-time prediction of the needle deflection, which can be more useful when presented visually than lowresolution ultrasound images. Also, the proposed model can be utilized for pre-surgery motion planning and optimized trajectory design in brachytherapy applications.

In this research, our experiments were performed only on a simple test phantom using synthetic materials. Further experiments in more realistic conditions, e.g., in-vitro tests using biological tissues are needed to further verify our conclusions. Also, velocity is the only input of the presented model. However, axial rotation of needle is another input that can give physicians control over needle trajectory. In future work rotation of beveled tip of the needle will be added to the model as another input.

\section{REFERENCES}

[1] N. Abolhassani, R. Patel, and M. Moallem, "Needle insertion into soft tissue: A survey," Medical Engineering \& Physics, vol. 29, no. 4, pp. $413-431,2007$.
[2] S. DiMaio and S. Salcudean, "Needle insertion modelling and simulation," in International Conference on Robotics and Automation, vol. 2, 2002, pp. 2098-2105.

[3] O. Goksel, S. Salcudean, and S. Dimaio, "3d simulation of needletissue interaction with application to prostate brachytherapy," Comput Aided Surgery, vol. 6, pp. 279-88, 2006.

[4] R. Webster, N. Cowan, G. Chirikjian, and A. Okamura, "Nonholonomic modeling of needle steering," in Experimental Robotics IX. Springer Berlin Heidelberg, 2006, vol. 21, pp. 35-44.

[5] R. Alterovitz, A. Lim, K. Goldberg, G. Chirikjian, and A. Okamura, "Steering flexible needles under markov motion uncertainty," in IEEE/RSJ International Conference on Intelligent Robots and Systems, 2005, pp. $1570-1575$.

[6] O. Goksel, E. Dehghan, and S. E. Salcudean, "Modeling and simulation of flexible needles," Medical Engineering \& Physics, vol. 31, no. 9, pp. 1069 - 1078, 2009.

[7] K. Yan, W. S. Ng, K.-V. Ling, Y. Yu, T. Podder, T.-I. Liu, and C. W. S Cheng, "Needle steering modeling and analysis using unconstrained modal analysis," in The First IEEE International Conference on Biomedical Robotics and Biomechatronics, 2006, pp. 87-92.

[8] T. Lehmann, M. Tavakoli, N. Usmani, and R. Sloboda, "Force-sensorbased estimation of needle tip deflection in brachytherapy," Journal of Sensors, vol. 2013, p. 10, 2013.

[9] A. Asadian, M. Kermani, and R. Patel, "A compact dynamic force model for needle-tissue interaction," in Annual International Conference of the IEEE Engineering in Medicine and Biology Society (EMBS), 2010, pp. 2292-2295.

[10] S. Misra, K. Reed, B. Schafer, K. Ramesh, and A. Okamura, "Mechanics of flexible needles robotically steered through soft tissue," Int. J. Rob. Res., vol. 29, no. 13, pp. 1640-1660, 2010.

[11] S. DiMaio and S. Salcudean, "Interactive simulation of needle insertion models," IEEE Transactions on Biomedical Engineering, vol. 52, no. 7, pp. 1167-1179, 2005.

[12] K. Yan, T. Podder, D. Xiao, Y. Yu, T.-I. Liu, C. Cheng, and W. Ng, "An improved needle steering model with online parameter estimator," International Journal of Computer Assisted Radiology and Surgery, vol. 1, no. 4, pp. 205-212, 2006

[13] A. Okamura, C. Simone, and M. O'Leary, "Force modeling for needle insertion into soft tissue," IEEE Transactions on Biomedical Engineering, vol. 51, no. 10, pp. 1707-1716, 2004.

[14] P. Hagedorn and A. DasGupta, Vibrations and Waves in Continuous Mechanical Systems. Wiley, 2007.

[15] L. Virgin, Vibration of Axially-Loaded Structures. Cambridge University Press, 2007.

[16] G. Genta, Vibration Dynamics and Control, ser. Mechanical Engineering Series. Springer, 2009.

[17] T. Johnson and P. Husbands, "System identification using genetic algorithms," in Parallel Problem Solving from Nature. Springer Berlin Heidelberg, 1991, vol. 496, pp. 85-89. 